\title{
EDITORIAL
}

\section{The need for regulatory science}

\author{
The quandary faced by regulators in deciding the fate of the controversial diabetes drug \\ rosiglitazone (Avandia; GlaxoSmithKline) illustrates the importance of investment and \\ innovation in the field of regulatory science.
}

In the middle of July this year, the 3-year controversy about the potential cardiovascular risks of rosiglitazone reached a pivotal point: the meeting of a US FDA advisory committee to provide recommendations on the future of the drug. The result was a $20-12$ vote in favour of rosiglitazone remaining on the market, with 17 of those voting in favour also recommending that warnings and/or restrictions on its usage should be strengthened. At the time of going to press, the FDA had yet to make its decisions based on the recommendations, but the history of the case so far illustrates some of the key issues for the future of drug regulation in the United States.

The controversy began in May 2007 with the publication of a meta-analysis of clinical trials of rosiglitazone suggesting that its use raised the risk of heart attacks ${ }^{1}$. Nevertheless, an FDA advisory committee meeting held in July 2007 voted that the overall benefit-risk profile of the drug supported its continued marketing. Since then, the final results of a randomized clinical trial known as RECORD, set up by GlaxoSmithKline specifically to investigate the potential cardiovascular risks of rosiglitazone, have been reported, which indicated that rosiglitazone does not increase the risk of cardiovascular hospitalization or cardiovascular death compared with standard glucose-lowering drugs ${ }^{2}$. Conversely, a recent observational study of patients in the United States receiving either rosiglitazone or pioglitazone (Actos; Takeda) - the other marketed drug in the same class - by staff at the FDA Office of Surveillance and Epidemiology concluded that rosiglitazone was associated with an increased risk of serious cardiovascular events compared with pioglitazone ${ }^{3}$.

The latest advisory committee meeting was convened to discuss all the available evidence on the cardiovascular safety of rosiglitazone, and to issue recommendations on two key questions. The first question, as noted above, was whether rosiglitazone should remain on the market. The second question was whether an ongoing randomized, controlled clinical trial directly comparing the cardiovascular safety of rosiglitazone and pioglitazone, known as TIDE, should continue. For this, the panel voted 19-11 that the trial should continue, although it has been argued that the trial is unethical given the evidence so far of the cardiovascular risks of rosiglitazone. Importantly, this second decision was made in the light of an initial report from the US Institute of Medicine on the ethical issues associated with trials such as TIDE, which recommended that a regulatory requirement to run such a trial should be based on the determination that neither the existing evidence nor new prospective observational studies can provide safety evidence sufficiently reliable for the FDA to make a sound policy decision.

Overall, the two main votes at the meeting highlight the scientific challenges of the rosiglitazone case. First, there is currently substantial uncertainty over the strength of the evidence indicating that rosiglitazone's benefit-risk profile does not support its continued marketing. Second, there are also considerable differences in opinion on the most appropriate strategy to resolve this uncertainty sufficiently to make a sound decision, including a debate on the relative merits of evidence from observational studies compared with randomized controlled trials. Helping to address such differences requires the continued development of regulatory science to incorporate the latest knowledge and tools, an area that has historically been lacking in funding but is now beginning to find new impetus under the current FDA leadership.

In this respect, the rosiglitazone case also highlights a concerning political dimension in the evolution of the FDA in general. For example, Representative Rosa DeLauro, the chair of the US government subcommittee responsible for funding the FDA, released a statement following the advisory meeting noting that she considered it "incomprehensible" that 20 committee members would vote to keep rosiglitazone on the market, and urging the FDA to withdraw it. So, as well as the quandary over the rosiglitazone decision in particular, the FDA has been faced with a broader key challenge: to show that it is prepared to make and explain such decisions based on the sound application of the best regulatory science available, independent of political interference. Politicians would be better advised to focus on providing the funding and environment to advance the field of regulatory science to assist regulators making these sensitive and complex decisions, rather than making their task harder through inappropriate political pressure.

\footnotetext{
. Nissen, S. E. \& Wolski, M. P. H. N. Engl. J. Med. 356, 2457-2471 (2007).

. Home, P. D. et al. Lancet 373, 2125-2135 (2009)

3. Graham, D. J. et al. JAMA 28 Jun 2010 (doi: 10.1001/jama.2010.920).
} 